\title{
ANÁLISE DO COMPORTAMENTO DO Preço dA SÉRIE DE CANA-DE-AÇÚCAR
}

\section{RESUMO}

O presente trabalho tem como objetivo estudar o comportamento das flutuações de preço da série da cana-de-açúcar. Trata-se de um estudo de caso quantitativo, cujos dados foram obtidos no site do Instituto de Economia Agrícola do Estado de São Paulo e perfazem 159 observações mensais do preço da cana-de-açúcar, abrangendo o período entre janeiro de 1995 e março de 2008. Os resultados evidenciaram a existência de tendência na série e não confirmaram a existência de sazonalidade significativa, mas apenas uma componente sazonal. Entre os três modelos propostos, o melhor foi o ARIMA $(1,1,0)(0,0,1)$ com duas intervenções. A primeira intervenção ocorreu por volta de agosto de 2006, e a segunda, em maio de 2007. Tanto a primeira quanto a segunda proporcionaram uma grande queda no preço da cana, principalmente em virtude da queda dos produtos provenientes da cana, tais como o álcool e o açúcar.

Palavras-chaves: Ajustamento. ARIMA. Cana-de-açúcar. Sazonalidade. Tendência.

\section{ABSTRACT}

This work aims to study the behavior of price fluctuations in the series of cane sugar. This is a case study in which quantitative data were obtained from the site of the Office of Agricultural Economics of the State of Sao Paulo and is composed by 159 monthly observations make up the price of cane sugar, covering the period from January 1995 to March 2008. The results showed the existence of trend in the series while not confirmed the existence of significant seasonality, only a seasonal component. Among the three proposed model was the best ARIMA $(1,1,0)(0,0,1)$ with two interventions. The first intervention occurred around August 2006 and the second refers to May 2007. Both the first and the second provided a large drop in the price of cane. This was mainly due to the decrease of products from sugarcane, such as alcohol and sugar.

Keywords: Adjustment. ARIMA. Cane sugar. Seasonality. Trend.

\author{
Pedro Luiz Costa Carvalho \\ Thelma Sáfadi \\ Luiz Eduardo Gaio Correio
}

Recebido em 08/outubro/2010 Aprovado em 30/novembro/2011 


\section{INTRODUÇÃO}

As séries temporais referem-se a conjuntos de dados ordenados no tempo, podendo ser relacionados à economia, em geral, por essa série. Dessa forma, modelos de análise de séries temporais são eventualmente utilizados para o estudo de dados dessa natureza. A característica mais importante desse tipo de dados é que as observações vizinhas são dependentes, daí o interesse de modelar essa dependência neste artigo. Em modelos de regressão, por exemplo, a ordem das observações é irrelevante para a análise, mas em séries temporais a ordem dos dados é crucial.

Entre os objetivos das análises de séries temporais destacam-se: a investigação do mecanismo gerador da série; a realização de previsões de valores futuros de curto e longo prazo; a descrição do comportamento da série, com verificação gráfica de existências de tendências, ciclo e variações sazonais; busca de periodicidade nos dados (Morettin \& Toloi, 2004).

As séries econômicas e em especial as de produtos alimentícios são, em sua grande maioria, afetadas pelos eventos sazonais, pelas tendências, pelos fatores exógenos e pelas intervenções governamentais, que podem dificultar a interpretação das flutuações dos preços das séries. Assim, a cana-de-açúcar, uma das mais importantes culturas para o País, tem seu preço influenciado por esses fatores na forma de controle do Estado, de aumento rápido da demanda, de aumento das exportações, de controle de preços, e de subsídios. Portanto, a correta identificação e mensuração desses fatores na série cana-de-açúcar pode ajudar a analisar perfeitamente as variações em seu preço.

Nesse contexto, o presente trabalho vem propor um modelo matemático que considere alguns dos fatores mais influentes nas flutuações do preço da canade-açúcar. Além disso, a pesquisa é uma oportunidade para o estudo do comportamento do preço da cultura em questão, com maior tendência de crescimento no mercado interno bem como no mercado externo.

\section{REFERENCIAL TEÓRICO}

\subsection{Mercado de Cana-de-Açúcar}

Historicamente a cana-de-açúcar é um dos principais produtos agrícolas do Brasil, sendo cultivada desde a época da colonização. Do seu processo de industrialização obtêm-se como produtos o açúcar, nas suas mais variadas formas e tipos, o álcool (anidro e hidratado), o vinhoto e o bagaço (Moraes \& Shikida, 2002 e Alves, 2002).

Na safra 1999/00, o Brasil produziu e moeu 300 milhões de toneladas de cana-de-açúcar, 381 milhões de sacas de $50 \mathrm{~kg}$ de açúcar e mais de 12 milhões de $\mathrm{m}^{3}$ de álcool anidro e hidratado. Já em 2007, a produção de etanol atingiu 21 bilhões de litros, dos quais $90 \%$ foram absorvidos pelo consumo interno. Essa tendência deverá manter seu crescimento até 2012, mas a demanda de exportação, que registrou 3,5 bilhões de litros em 2007, também deverá crescer muito nos próximos anos. De acordo com a União da Indústria de Cana-de-Açúcar (UNICA), a produção de etanol no Brasil deverá chegar a 65,3 bilhões de litros até 2020/21.

Dessa forma, o Brasil, hoje, é líder do mercado mundial de cana e conta com 7 milhões de hectares de cultura de cana-de-açúcar, área que deverá crescer muito nos próximos anos, pois o setor sucroalcooleiro brasileiro despertou o interesse de diversos países, principalmente pelo baixo custo de produção de açúcar e álcool. Este último tem sido cada vez mais importado por nações de primeiro mundo, que visam reduzir a emissão de poluentes na atmosfera e a dependência de combustíveis fósseis.

Entretanto, conforme relata Masieiro \& Lopes (2008), embora o Brasil seja líder na produção mundial de cana-de-açúcar, é necessário aumentar a capacidade para atender às exigências mundiais e internas de mercado, com foco no negócio de biocombustíveis. A demanda interna por álcool vem crescendo desde o lançamento, em 2002, dos veículos bicombustíveis. A venda de carros movidos a álcool e/ou bicombustíveis passou de 10.942 unidades, em 1999, para 379.328, em 2004, segundo a Associação Nacional de Fabricantes de Veículos Automotores (ANFAVEA). A demanda externa desse produto cresce a cada ano em virtude da necessidade de redução da emissão de poluentes na atmosfera, de tal forma que o Brasil exportou 2,4 bilhões de litros de álcool em 2004, com crescimento 
superior a $200 \%$.

Outro fator importante, relatado por Paulilio et al. (2007), é a vitória brasileira contra os subsídios europeus ao açúcar na Organização Mundial do Comércio(OMC). No mercado internacional, a cotação do açúcar na Bolsa de Nova Iorque vem acumulando alta, rompendo a barreira dos dez centavos de dólar/ libra peso, a maior cotação nos últimos sete anos.

Devido à grandeza dos números do setor sucroalcooleiro no Brasil, não se pode tratar a canade-açúcar apenas como mais um produto, mas, sim, como o principal tipo de biomassa energética, base para todo o agronegócio sucroalcooleiro, representado por 350 indústrias de açúcar e álcool e 1.000 .000 de empregos diretos e indiretos em todo o Brasil.

\subsubsection{Formação do preço da cana-de-açúcar}

Durante a intervenção estatal no setor sucroalcooleiro, o preço sempre foi regulado de modo a assegurar a rentabilidade e, ao mesmo tempo, possibilitar a contenção do processo inflacionário. Dessa forma, os preços eram fixados tomando como referencial o valor constante de planilhas de custo de produção, acrescido de montante que representasse o lucro da atividade (Alves, 2002).

Ainda de acordo com Alves (2002), com a desregulamentação do setor, estabeleceram-se condições mais competitivas, visto que os preços dos produtos finais (açúcar e álcool) e da matéria-prima passaram a ser determinados de acordo com as regras de livre mercado. Sendo assim, o preço da cana-deaçúcar não é mais determinado pelo governo desde a safra 1998/99. Diante disso, surgiram entidades com o intuito de organizar o setor, que se encontrava durante décadas sob intervenção estatal.

No Estado de São Paulo, constituiu-se um grupo formado por representantes dos produtores de cana, representados pela Organização de Plantadores de Cana da Região Centro-Sul do Brasil (ORPLANA), e industriais, representados pela União da Agroindústria do Açúcar e do Álcool do Estado de São Paulo (UNICA), com o objetivo de desenvolver um novo sistema para a remuneração da cana-de-açúcar. Assim, surgiu o Conselho de Produtores de Cana, Açúcar e Álcool de São Paulo (CONSECANA) para programar a nova sistemática.

O modelo atual de pagamento de cana é denominado Sistema de Remuneração da Tonelada de Cana pela Qualidade/CONSECANA, que considera, para efeito de determinação do valor da tonelada da cana-de-açúcar, a quantidade de Açúcar Total Recuperável (ATR) contida na matéria-prima, entregue na unidade de processamento, e o preço do quilograma do ATR (Alves, 2002).

O preço do quilograma do ATR é determinado em função do preço do açúcar, nos mercados interno estadual (branco) e externo (branco e VHP), do preço do álcool anidro e hidratado (carburante e industrial, nos mercados interno estadual e externo), livre de impostos ou frete, do 'mix' de produção de cada unidade industrial, ou seja, da quantidade produzida de açúcar e álcool, e da participação da matéria-prima nos custos de produção do açúcar e do álcool.

No início do ano-safra, o CONSECANA divulga o 'mix' de produção para o Estado de São Paulo, que será utilizado no cálculo do preço mensal estimado do quilograma do ATR, juntamente com os preços dos produtos finais praticados no mercado e a participação percentual da matéria-prima nos custos de produção.

$\mathrm{O}$ fornecedor entrega a cana na unidade industrial, onde é calculado o total de ATR, e recebe um adiantamento mensal, de cerca de $80 \%$ do valor, denominado nota de entrega. Ao final do ano-safra, é feito o ajuste final com base no 'mix' de produção efetivamente realizado pela unidade compradora e pela quantidade de ATR entregue pelo fornecedor.

\subsection{Modelos ARIMA}

Os modelos autorregressivos foram desenvolvidos por Yule em 1926, enquanto os modelos de médias móveis foram desenvolvidos por volta de 1937. No decorrer dos anos, muitos autores ajudaram a desenvolver essa técnica de análise, dos quais se podem citar Winters (1960), Box \& Cox (1964), Jenkins \& Watts (1968), Box \& Tiao (1975), Priestley (1981), Box et al. (1994) e Morretin \& Toloi (2004). Entretanto, Box \& Jenkin desenvolveram, em 1970, uma metodologia para modelos de previsão largamente utilizada. Essa metodologia consiste em ajustar modelos autorregressivos integrados de médias 
móveis, ARIMA, a um conjunto de dados.

\subsubsection{Modelo autorregressivo (AR)}

De acordo com Box et al. (1994) e Morretin \& Toloi (2004), sejam $\bar{Z}_{t}=Z_{t}-\mathrm{m}$ os desvios em relação a $m$. Então,

$$
\bar{Z}_{t}=\mathrm{f}_{1} \bar{Z}_{t-1}+\mathrm{f} \bar{Z}_{t-2}+\ldots+\mathrm{f}{ }_{p} \bar{Z}_{t-p}+a_{t}
$$

É um processo autorregressivo de ordem $\mathrm{p}$, dentado por AR(p) (Morettin e Toloi, 2004). Em outras palavras, a série é representada por uma soma ponderada de " $p$ " observações anteriores da série mais um termo aleatório. Definindo-se o operador autorregressivo de ordem " $p$ " por:

$\mathrm{f}(B)=1-\mathrm{f}_{1} B-\ldots-\mathrm{f}_{p} B^{p}$

em que $B^{p} Z_{t}=Z_{t-p}$ é o operador de retardo.

Pode-se escrever:

$\mathrm{f}(B) \bar{Z}_{t}=a_{t}$

em que " $a_{t}$ " é resíduo (ou ruído).

\subsubsection{Modelo de médias móveis}

Morretin \& Toloi (2004) definem um processo

$\bar{Z}=a_{t}-\mathrm{q}_{1} a_{t-1}-\mathrm{q}_{2} a_{t-2}-\ldots-\mathrm{q}_{q} a_{t-q}$

em que a série é vista como uma soma ponderada de " $q$ ” observações anteriores do ruído, como um processo de médias móveis de ordem " $q$ ", denotado por MA(q). Definindo-se o operador de médias móveis e ordem " $q$ " por:

$\mathrm{q}(B)=1-\mathrm{q}_{1} B-\mathrm{q}_{2} B^{2}-\ldots-\mathrm{q}_{q} B^{q}$

pode-se escrever

$\bar{Z}_{t}=\mathrm{q}(B) a_{t}$

2.2.3 Modelo misto autorregressivo e de médias móveis (ARMA)
Éomodeloque incluitanto termos autorregressivos como termos de médias móveis, sendo denotado por ARMA (p,q) Morretin \& Toloi (2004):

$\bar{Z}_{t}=\mathrm{f}_{1} \bar{Z}_{t-1}+\ldots+\mathrm{f}_{p} \bar{Z}_{t-p}+a_{t}-\mathrm{q}_{1} a_{t-1}-\ldots-\mathrm{q}_{q} a_{t-q}$

ou

$$
\mathrm{f}(B) \bar{Z}_{t}=\mathrm{q}(B) a_{t}
$$

2.2.4 Modelos autorregressivos integrados de médias móveis (ARIMA)

De acordo com Morretin \& Toloi (2004) se $W_{t}=\Delta^{d} Z_{t}$ for estacionária, pode-se representar por $W_{t}$ um modelo ARMA $(\mathrm{p}, \mathrm{q})$, ou seja,

$\mathrm{f}(B) \bar{Z}_{t}=\mathrm{q}(B) a_{t}$

Se $W_{t}$ for uma diferença de $Z_{t}$, então $Z_{t}$ é uma integral de $W_{t}$; daí tem-se que $Z_{\mathrm{t}}$ segue um $\mathrm{m}$ o $\mathrm{d}$ e 1 o autorregressivo, integrado, de médias móveis, ou modelo ARIMA,

$\mathrm{f}(B) \Delta^{d} \bar{Z}_{t}=\mathrm{q}(B) a_{t}$

de ordem $(\mathrm{p}, \mathrm{d}, \mathrm{q})$.

\subsubsection{Modelos com intervenção}

Por uma intervenção entendemos a ocorrência de algum tipo de evento em dado instante de tempo T, conhecido a priori. Tal ocorrência pode manifestar-se por um intervalo de tempo subseqüente, que afeta temporária ou permanentemente a série em estudo (Morettin \& Toloi, 2004). De acordo com Box \& Tiao (1975), a análise de intervenção tem por objetivo avaliar o impacto de tal evento no comportamento da série. Uma classe geral de modelos, que leva em conta a ocorrência de múltiplas intervenções, é dada por:

$Z_{t}=\sum_{j=1}^{k} \mathrm{u}_{j}(B) X_{j, t}+N_{t}$ 
Em que $v_{i}(B)$ é da função de transferência da forma:

$$
v_{i}(B)= \begin{cases}\frac{\omega(i)}{1-\delta_{i} B} & (1) \\ \mathbf{u} & (2) \\ \omega(i) & \text { em que }\left|\delta_{i}\right|<1\end{cases}
$$

$\delta_{\mathrm{i}}$ é o efeito de intervenção até atingir um novo nível na série temporal. A função de transferência $v_{i}(B)$, do tipo (1), é considerada uma intervenção gradual permanente, e a do tipo (2) é tida como igual a uma intervenção abrupta permanente (Box \& Tiao, 1975).

\section{MATERIAL E MÉTODOS}

O presente trabalho adotou o estudo de caso como metodologia básica. De acordo com Patton (1990), Sampieri et al. (1991) e Santos (1991), o estudo de caso pode proporcionar um retrato válido sobre o programa, uma boa base para as pessoas entenderem o que está acontecendo, bem como bases sólidas para entender o desenrolar das ações programadas.

Quanto à concepção metodológica, utilizou-se um estudo quantitativo, visto que ela permite analisar, por meio de um conjunto limitado de questões, as relações de um grupo relativamente grande de informações, facilitando a comparação e o tratamento estatístico dos dados. Selltiz et al. (1974) ressaltam que esses estudos supõem que as medidas sejam, além de precisas e confiáveis, construídas com base em modelos que permitam demonstrar relações de causalidade, sendo, por isso mesmo, construídos de acordo com a lógica das explicações científicas, isto é, por meio de requerimentos de verificação lógica, tal como a consistência entre as proposições que integram o modelo explicativo e de verificação empírica, a exemplo da correspondência das proposições do modelo com a realidade empírica.

Dessa forma, a definição do problema de pesquisa foi motivada pela percepção da grande mudança que vem ocorrendo na agricultura e no preço da cana-deaçúcar depois do aumento da demanda pelo álcool. Particularmente, esta pesquisa procurou analisar as variações no preço mensal da tonelada da cana-deaçúcar, no período entre janeiro de 1995 e março de 2008. Os dados para a pesquisa foram obtidos no Instituto de Economia Agrícola do Estado de São Paulo, por meio do site www.iea.sp.gov.br, e analisados estatisticamente a fim de ajustar modelos matemáticos para explicar as principais características envolvidas na movimentação do preço/tonelada mensal da canade-açúcar.

O processo de análise consiste, primeiramente, em verificar na série original de preço a existência das componentes tendência e sazonalidade. A tendência é verificada por meio do gráfico da função de autocorrelação da série original e comprovada pelo teste do sinal de Cox-Stuart. Para mais informações sobre o teste, ver Morretin \& Toloi (2004).

Para a verificação da sazonalidade, utilizou-se a decomposição espectral da série de preço em uma série de Fourier. Nessa análise, é como se se colocasse a série em um prisma com a finalidade de identificar o tamanho das ondas e a importância dos componentes cíclicos. Dessa forma, flutuações sazonais de diferentes comprimentos podem ser identificadas e testadas (Jenkins e Watts, 1968; Priestley, 1981; Shumway, 1988; Wei, 1989). Um teste bastante utilizado é o denominado G, de Fisher. De acordo com o teste, se o valor de "G" - calculado por meio do periodograma - for maior do que o valor de "Z", então a série apresenta periodicidade. A estatística do teste pode ser descrita da forma:

$$
z=1-\sqrt[n-1]{\frac{\mathrm{a}}{n}} g=\frac{\max I_{j}^{(N)}}{\sum_{j=1}^{\left[\frac{N}{2}\right]} I_{j}^{(N)}}
$$

em que:

$\mathrm{I}=$ maior valor do periodograma,

$\mathrm{N}=$ tamanho da série dividido por 2,

$\alpha=$ nível de significância de $95 \%$.

De acordo com Morettin \& Toloi (2004), após a identificação e a eliminação desses efeitos por meio de diferenças, inicia-se a identificação e a estimação dos parâmetros ARMA. Isso é feito por meio da utilização 
do gráfico da função de autocorrelação — FAC — e do gráfico da função de autocorrelação parcial FACP. Enquanto o primeiro mostra a ordem do MA, o segundo mostra a ordem do AR.

Em seguida, verifica-se a possibilidade de os modelos escolhidos terem deixado os resíduos da série estacionários e não correlacionados, ou seja, um ruído branco, o que é feito observando-se o gráfico da função de autocorrelação dos resíduos e confirmando pelo teste de Box \& Pierce. O teste consiste em comparar o valor da estatística chi ${ }^{2}$ com o valor de $\mathrm{Q}$. Se o valor de $\mathrm{Q}$ for menor do que o valor de $\mathrm{chi}^{2}$, então a hipótese de ruído branco para os resíduos é aceita. A estatística de $\mathrm{Q}$ pode ser definida da seguinte forma:

$$
\text { (13) } Q(K)=n(n+2) \sum_{j=1}^{k} \frac{r_{j}^{2}}{(n-j)}
$$

Por fim, para verificar qual modelo é o melhor, utiliza-se o Critério de Informação de Akaike - AIC — e do Critério de Informação Bayesiano — BIC. O AIC e o BIC são dados pelas estatísticas:

$$
\begin{array}{r}
A I C(k, l)=\ln \left(\mathrm{s}_{k, l}^{2}\right)+2(k+l) / n \\
B I C(k, l)=\ln \left(\mathrm{s}_{k, l}^{2}+(k+l) \ln (n) / n\right.
\end{array}
$$

em que:

$\mathrm{K}$ e L = parâmetros do modelo,

$\ln \left(\mathrm{s}_{k, l}^{2}\right)=$ logarítmico da variância.

\section{RESULTADOS E DISCUSSÕES}

A Figura 1 demonstra o gráfico da série de preço da tonelada de cana-de-açúcar no período entre janeiro de 1995 e março de 2008. Ao observar o gráfico com mais cuidado, nota-se uma grande alteração na série, por volta da observação 55. Nessa época, o governo parou de controlar o preço da cana, deixando-o livre ao mercado. Desde então, ocorreu um crescimento no preço, que perdurou até meados de 2006, observação 135 a 140. Um dos principais fatores responsáveis por esse aumento foi a crescente demanda por carros a álcool, intensificada a partir de 2002. Contudo, desde meados de 2006 o preço sofreu uma grande queda, voltando aos patamares de 2003-2005.

A seguir, busca-se comprovar e mensurar as duas principais características presentes em séries de preços de produtos agrícolas: a tendência e a sazonalidade. Com relação à tendência, sua existência está clara no gráfico da Figura 1 e também pode ser observada pela Figura 2, a qual mostra a função de autocorrelação da série. De acordo com a Figura 2, os valores dos lags decaem lentamente para zero, característica de séries que possuem tendência. Para comprová-la, aplicou-se o teste do sinal, introduzido por Cox-Stuart. O resultado do teste veio confirmar o que era evidente: a série possui tendência crescente. Então, fez-se a primeira diferença da série a fim de eliminar esse efeito, conforme demonstrado pelo gráfico da Figura 3.

Veja-se que a série tornou-se estacionária após a diferença, ou seja, desenvolveu-se no tempo aleatoriamente, ao redor de uma média constante, refletindo alguma forma de equilíbrio estável. Por esse gráfico ainda é possível notar um topo e um fundo bem acentuado, que podem representar alguma forma de intervenção externa. Sua significância será estudada mais adiante.

Dessa forma, iniciou-se a análise da presença de sazonalidade na série. A sazonalidade é muito comum em séries de produtos agrícolas, que geralmente acontecessem na época da safra e da entressafra, em virtude de mudanças na oferta do produto dentro de uma mesma demanda. Assim, o preço tende a cair na safra e a subir na entressafra, o que caracteriza a sazonalidade. Para comprová-la, fez-se o teste de Fischer, em que se compara o valor $\mathrm{G}$ calculado com o valor de $Z$. Ao realizar o teste, o valor de $G$ calculado foi menor do que o de $\mathrm{Z}$, caracterizando, assim, a não existência de sazonalidade na série. Apesar de ser um produto agrícola, a série da canade-açúcar pode não ter apresentado sazonalidade identificável porque nos últimos anos a demanda pelo álcool foi crescente e movimentou toda a cadeia de cana, bem como a relação oferta/demanda.

Com as duas componentes analisadas, iniciou-se o ajuste de um modelo matemático que explicasse o comportamento da série. Para tanto, utilizou-se 
a série diferenciada para a análise, visto que ela se tornou estacionária. Conforme já mencionado, a metodologia para a análise está baseada nas modelagens ARIMA propostas por Box \& Jenkins. Com base nessa metodologia fez-se o gráfico da função de autocorrelação e autocorrelação parcial a fim de verificar as possíveis ordens do modelo. Os dois gráficos estão representados, respectivamente, pelas Figuras 4 e 5.

A função de autocorrelação (FAC), demonstrada pela Figura 4, sugere um MA(3) ou um MA(4), entre outros modelos. Não há uma definição clara de qual modelo se ajustaria mais fácil. Já o gráfico da função de autocorrelação parcial (FACP), demonstrado pela Figura 5, sugere um AR(1) ou um AR(2), no máximo. Além disso, ocorre na FACP dois lags múltiplos de 12, fora do nível de significância, o que pode indicar a necessidade de alguma ordem sazonal a ser ajustada, mesmo que o modelo como um todo não apresente sazonalidade.

Outra característica a ser estudada são os fundos e topos verificados no gráfico da primeira diferença da série, conforme mostrado pela Figura 3. O gráfico sugere a análise de três topos (observações em torno de 70,100 e 140) e quatro fundos (observações em torno de 62, 90, 110 e 150).

Sendo assim, várias combinações de modelos foram testadas visando sempre o melhor modelo. Testaram-se, também, todos os topos e fundos mencionados. Entre todos os modelos, selecionaramse três que explicam melhor a série. Os três modelos com seus parâmetros estão resumidos na Tabela 1 .

É importante ressaltar que para os modelos 1 e 2 adicionou-se uma componente sazonal, representada pela letra Q. No modelo 3, essa variável não foi considerada. Isso foi feito para verificar e testar quão importante é essa variável no ajuste como um todo.
Além disso, todas as variáveis dos modelos foram significativas. Das 7 possíveis intervenções (3 topos e 4 fundos) apenas 2 foram consideradas, visto que sua significância apareceu em quase todos os modelos testados. Dessa forma, a primeira intervenção ocorreu de forma abrupta e permanente por volta de agosto de 2006, o que causou um grande recuo no preço. Já a segunda intervenção ocorreu por volta de maio de 2007 e foi influenciada pela primeira, também causando uma diminuição dos preços, contudo de forma mais gradual.

Essa grande queda ocorrida no preço da canade-açúcar e detectada pelas duas intervenções foi motivada pela diminuição dos produtos provenientes da cana. A queda dos preços do açúcar foi causada pelo excesso de oferta no mercado internacional, resultado da recomposição da safra 2006/2007 dos países tradicionalmente grandes produtores, como Índia e Austrália. Após duas safras prejudicadas por problemas climáticos, esses países recuperaramse na safra 2006/2007 e apresentaram índices de produção e produtividade em patamares históricos. Adicionalmente, os altos preços internacionais do açúcar também incentivaram esses países a retomar seus níveis históricos de produção. Com relação ao álcool, a maior produção, aliada à queda do preço do barril do petróleo naquela época, pressionou a queda dos preços. Para combater o excesso do produto no mercado e alavancar seus valores, o governo aumentou, no ano passado, de $20 \%$ para $23,5 \%$ a adição de álcool à gasolina, tentando enxugar o mercado em cerca de 300 mil litros de álcool anidro (CNA, 2008).

Em seguida e antes de comparar a qualidade de ajuste dos modelos, submeteram-se cada um ao teste de Box \& Pierce para verificação da existência de correlação dos resíduos. Para um bom ajuste, os resíduos não podem estar correlacionados, ou seja,

Tabela 1 - Resumo das características dos três modelos escolhidos

\begin{tabular}{|c|c|c|c|c|c|c|c|c|c|c|c|}
\hline \multirow{3}{*}{ Modelo } & \multirow{2}{*}{\multicolumn{2}{|c|}{ Dif. }} & \multicolumn{5}{|c|}{ Parâmetros ARMA } & \multirow{2}{*}{\multicolumn{3}{|c|}{ Intervenções }} & \multirow{3}{*}{ MS } \\
\hline & & & p & & & $\mathbf{P}$ & Q & & & & \\
\hline & D & D & 1 & 1 & 2 & 1 & 1 & Om(1) & $\operatorname{Om}(2)$ & $\operatorname{Del}(2)$ & \\
\hline 1 & 1 & 0 & - & $-0,315$ & $-0,182$ & - & 0,225 & 3,869 & $-1,895$ & 0,852 & 0,5446 \\
\hline 2 & 1 & 0 & 0,350 & - & - & - & 0,207 & 3,976 & $-1,889$ & 0,823 & 0,5438 \\
\hline 3 & 1 & 0 & 0,369 & - & - & - & - & 3,889 & $-2,16$ & 0,839 & 0,5626 \\
\hline
\end{tabular}

d: diferença tendência, D: diferença sazonal, p: operador autorregressivo; q: operador de médias móveis; P: operador autorregressivo sazonal; Q: operador de médias móveis sazonal; MS: variância.

Fonte: elaborada pelos autores. 
todos os dados, ou mais de $95 \%$ deles, têm de estar dentro do intervalo de confiança. Sendo assim, o modelo 1 apresentou o valor de chi $^{2} 44,98$ contra o valor de Q de 39,49; o modelo 2 apresentou o valor de 46,19 para o chi ${ }^{2}$ contra 43,21 para o valor de Q; por fim, o modelo 3 apresentou o valor de 46,19 para o chi ${ }^{2}$ contra o valor de 50,59 de Q.

Observe-se que apenas no modelo 3 o valor de Q está maior do que o valor da estatística $\mathrm{chi}^{2}$, o que significa que o resíduo do modelo 3 ainda está um pouco correlacionado, provavelmente devido à retirada da componente sazonal Q. O fato de os outros dois modelos possuírem uma componente sazonal não significa a existência de sazonalidade, mas, sim, a presença de um resquício de sazonalidade sem força suficiente para influenciar a variação do preço como um todo. Dessa forma, o modelo 3 não é tão adequado para explicar o comportamento dos preços da série de cana-de-açúcar.

A análise final tem por objetivo comparar qual dos três modelos de fato possui o melhor ajustamento, utilizando-se o Critério de Informação de Akaike (AIC) e o critério de Informação Bayesiano (BIC). De acordo com esses critérios, aquele que apresentar menor valor é o melhor modelo. A Tabela 2 mostra o valor de cada critério para os três modelos.

TABELA 2 - Comparativo entre os modelos

\begin{tabular}{ccc}
\hline & AIC & BIC \\
\hline Modelo 1 & $-0,54481$ & $-0,4483$ \\
Modelo 2 & $-0,55886^{*}$ & $-0,48165^{*}$ \\
Modelo 3 & $-0,52487$ & $-0,44767$ \\
\hline
\end{tabular}

Nota: * representa o melhor modelo.

Fonte: elaborada pelos autores.

De acordo com os dois testes, o melhor modelo foi o 2, em comparação com os outros 2 modelos. Esse resultado é plausível, visto que o modelo 2, em comparação com o 1, possui menor número de parâmetros, ou seja é mais parcimonioso. Já em comparação com o modelo 3, além do menor número de parâmetros leva-se em conta também a não inclusão da componente sazonal, o que deixou o modelo ainda correlacionado.

Corroborando a qualidade de ajustamento do modelo 2, mostra-se, a seguir, o seu gráfico da função de autocorrelação dos resíduos. Note-se que de acordo com o gráfico praticamente não há valores de lags fora do intervalo de confiança, confirmando, assim, a característica de ruído branco, ou seja, a não correlação dos dados.

\section{CONCLUSÃO}

O presente trabalho possibilitou estudar melhor o comportamento dos preços da série de cana-deaçúcar, demonstrando quais fatores a influenciam. Ficou evidente a grande pressão que a demanda pelo álcool, motivada pela entrada dos carros flex no mercado, causou no preço da tonelada de cana. Desde a desregulamentação do governo, no ano de 1999, o preço sofreu um grande aumento, contraindo-se somente no último ano.

De acordo com os resultados encontrados, podese verificar que a série apresentou tendência crescente, mas não apresentou sazonalidade, fato incomum em séries agrícolas largamente influenciadas pelas estações do ano e por fatores culturais. Considerando, então, apenas a tendência como componente inicial, ajustaram-se três modelos à série de preços.

Nesse momento, a dificuldade de ajuste foi muito grande, de forma que praticamente nenhum modelo, nem mesmo os que sofreram intervenções, foi capaz de ajustar de forma satisfatória a série. Testouse, assim, a alternativa de adicionar uma componente sazonal ao modelo com intervenções, com base na observação dos gráficos da função de autocorrelação. Devido a essa componente, foi possível ajustar dois modelos (1 e 2) satisfatórios à série, além de um modelo (3) muito próximo do satisfatório, mas sem a componente sazonal. Dessa forma, ficou claro que apesar de não possuir sazonalidade significativa ela está presente de forma bem discreta. Além disso, para todos os modelos, consideraram-se duas intervenções, uma na observação 140 representando o período de agosto de 2006, e outra na observação 159 representando o período de maio de 2007.

Para a definição do melhor ajuste matemático à série, três modelos foram submetidos aos Critérios de Informação de Akaike (AIC) e de Bayesiano 
(BIC). Entre os três modelos, o pior, conforme já era esperado, foi o modelo 3, sem a componente sazonal. Em contrapartida, o melhor modelo foi o 2 “" $(1,1,0)$ $(0,0,1)$ com duas intervenções". Apesar do modelo 1 também ser satisfatório, o modelo 2 o superou, pois foi mais parcimonioso, ou seja, apresentou menor número de variáveis explicativas.

Portanto, recomenda-se a utilização do modelo para se entender melhor as particularidades da série do preço da cana-de-açúcar.

Entre os beneficiários desta pesquisa estão os produtores rurais, as associações comerciais, os órgãos públicos, os sistemas educacionais e os consumidores de uma forma geral.

\section{REFERÊNCIAS}

ALVES, L. R. A. Transmissão de preços entre produtores do setor sucroalcooleiro do Estado de São Paulo. 2002. 107 f. Dissertação (Mestrado em Economia Aplicada) Escola Superior de Agricultura Luiz Queiroz, Universidade de São Paulo, Piracicaba.

Associação Nacional dos Fabricantes de Veículos Automotores - ANFAVEA. <www.anfavea.com.br>.

BOX, G. P. E.; JENKINS, G. M.; REINSEL, G. Time Series Analysis: forecasting and control. Third Edition. Englewood Cliffs: Prentice Hall, 1994, 592p.

BOX, G. E. P.; TIAO, G. C. Intervention analysis with applications to economic and environmental problems. Journal of the American Statistical association, vol. 70, pp.70-79, 1975.

DEPARTAMENTO DE COMUNICAÇÃO DACNA; Dados do Balanço 2006 e Perspectivas 2007 para agropecuária brasileira divulgados pela Confederação da Agricultura e Pecuária do Brasil (CNA) - (Texto 6/16), Alimento Seguro, 2008.

Instituto de Economia Agrícola do Estado de São Paulo IEA $-<$ www.iea.sp.gov.br $>$.

JENKINS, G. M.; WATTS, D. G. Spectral analysis and its applications. San Francisco: Holden-Day, 1968. 525p.

MASIEIRO, G.; LOPES, H. Etanol e biodiesel como recursos energéticos alternativos: perspectivas da América Latina e da Ásia. Revista Brasileira de Política Internacional, Brasília, v. 51, n. 2, p. 60-79, jul./ dez. 2008.
MENTZ, R. P.; MORETTIN, P. A.; TOLOI, C. M. C. Bias correction for the ARMA $(1,1)$ Model. Estatística, vol. 53, n. 160,161 , pp. 1-40, 2001.

MORAES, M. A. F. D. de; SHIKIDA, P. F. A. (Org.). Agroindústria canavieira no Brasil: evolução, desenvolvimento e desafios. São Paulo: Atlas, 2002. 368 p.

MORETTIN, P. A.; TOLOI, C.M.C. Análise de séries temporais. São Paulo: Edgard Blucher. 2004. 535p.

SAMPIERI, R. H.; COLLADO, C. F.; LUCIO, P. B. Metodología de la investigación. México: McGraw-Hill, $1991.896 \mathrm{p}$.

SANTOS, J. V. T. A construção da viagem inversa. Ensaio sobre a investigação nas ciências sociais. Cadernos de Sociologia. Porto Alegre: v. 3, n. 3, p. 55-88, jan./jul.1991.

Organização de Plantadores de Cana da Região Centro-Sul do Brasil - OPLANA. <http://www.orplana.com.br/>.

PATTON, M. Q. Qualitative evaluation and research methods. Londres: Sage, 1990. 338p.

PAULILLO, L. F.; VIAN, C. E. de F.; SHIKIDA, P. F. A.; MELLO, F. T. de. Álcool combustível e biodiesel no Brasil: quo vadis? Revista de Economia e Sociologia Rural, Brasília, v. 45, n. 3, p. 531-565, jul./set. 2007.

PRIESTLEY, M. B. Spectral analysis and time series Academic Press Inc, London, England, 1981. 890p.

PRIESTLEY, M. B. Non-linear and Non-stationary Time Series Analysis. New York: Academic Press, 1988. 227p.

SAID, S. E.; DICKEY D. A. Hypothesis testing in $\operatorname{ARIMA}(p, 1, q)$ models. Journal of the American Statitical Association, vol. 80, n. 1 pp. 369-374, 1985.

SEATER, J. J. World temperature: trend uncertainties and their implications for economic policy. Journal of Business and Economic Statistics, vol. 11 pp. 265-277, 1993.

SELLTIZ, C. et al. Métodos de pesquisa nas relações sociais, 4a. ed. São Paulo: EDUSP, 1974. 150p.

SHUMWAY, R.H. Applied statistical time series analysis. Englewood Cliffs, NJ: Prentice Hall, 1988. 384p.

União da Agroindústria do Açúcar e do Álcool do Estado de São Paulo - ÚNICA. www.única.com.brt/.

WEI, W.W. Time series analysis: Univariate and multivariate methods. New York: Addison-Wesley, 1989. 478p.

WINTERS, P. R. Forecasting Sales by exponentially weighted moving average.

Management Science, vol. 6, n. 1 pp. 324-342, 1960. 


\section{APÊNDICE A}

FIGURA 1 - Preço médio mensal recebido pelos agricultores em Toneladas no período entre janeiro de 2005 e março de 2008

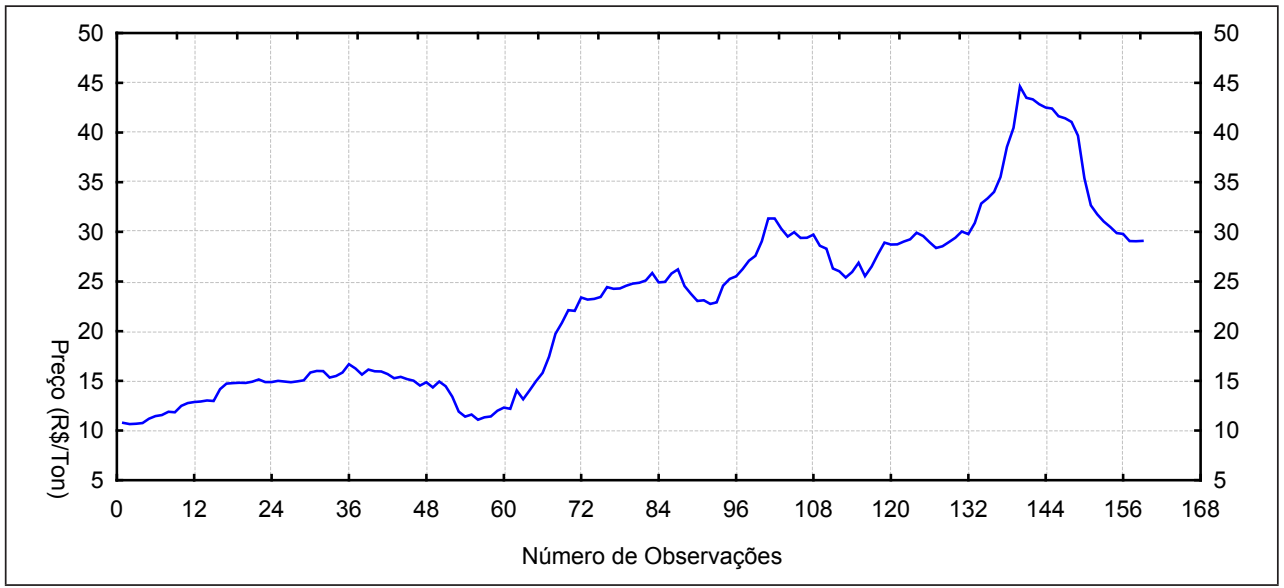

Fonte: <www.iea.sp.gov.br $>$.

FIGURA 2 - Função de autocorrelação da série original de preço

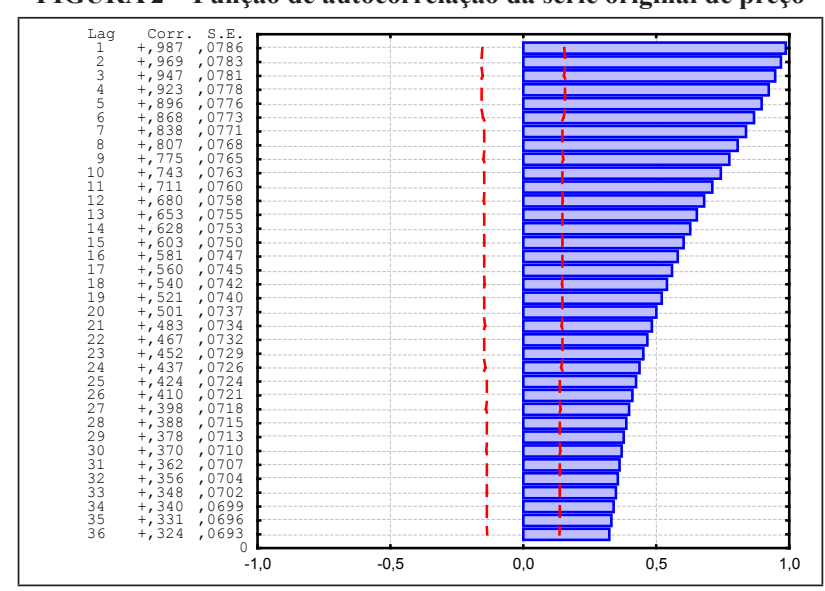

Fonte: elaborada pelos autores.

FIGURA 4 - Função de autocorrelação

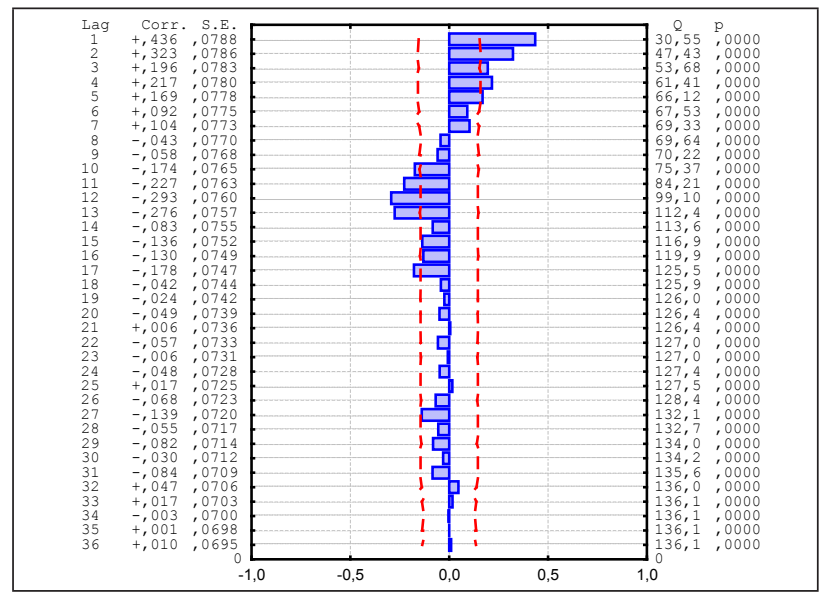

Fonte: elaborada pelos autores.
FIGURA 3 - Gráfico da $1^{\text {a }}$ diferença da série

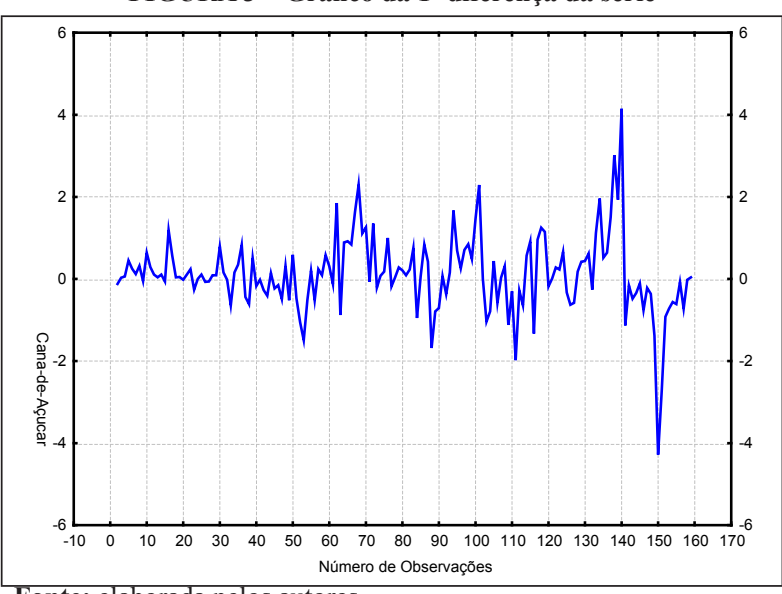

Fonte: elaborada pelos autores.

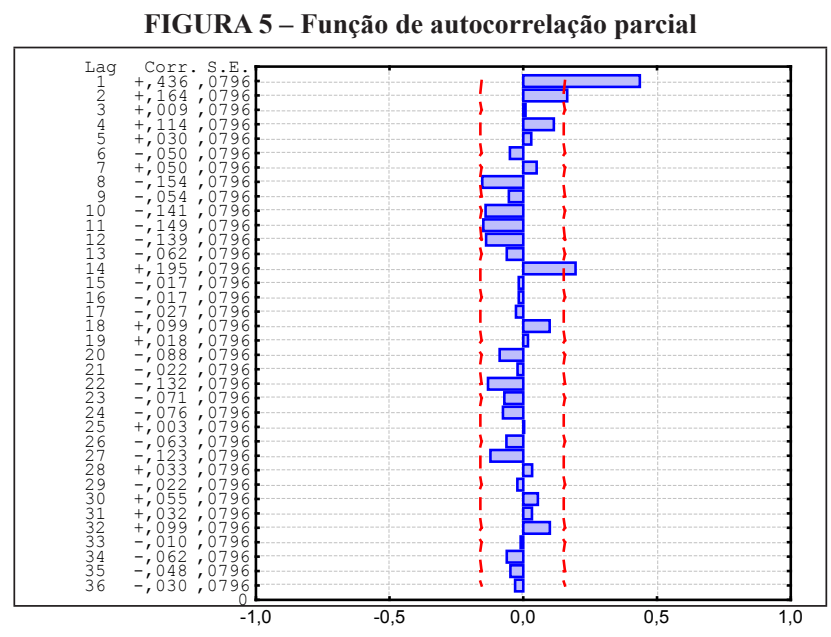

Fonte: elaborada pelos autores 